\title{
Multicritical Fermi Surface Topological Transitions
}

\author{
Dmitry V. Efremov, ${ }^{1, *}$ Alex Shtyk, ${ }^{2, *}$ Andreas W. Rost,${ }^{3,6, \uparrow}$ Claudio Chamon, ${ }^{4}$ \\ Andrew P. Mackenzie, ${ }^{3,5}$ and Joseph J. Betouras $\circledast^{1, \$}$ \\ ${ }^{1}$ Department of Physics and Centre for the Science of Material, Loughborough University, Loughborough LE11 3TU, United Kingdom \\ ${ }^{2}$ Department of Physics, Harvard University, Cambridge, Massachusetts 02138, USA \\ ${ }^{3}$ School of Physics and Astronomy, University of St Andrews, North Haugh, St Andrews, Fife KY16 9SS, United Kingdom \\ ${ }^{4}$ Department of Physics, Boston University, Boston, Massachusetts 02215, USA \\ ${ }^{5}$ Max Planck Institute for Chemical Physics of Solids, Noethnitzer Strasse 40, 01187 Dresden, Germany \\ ${ }^{6}$ Max Planck Institute for Solid State Research, Heisenbergstrasse 1, 70569 Stuttgart, Germany
}

\section{(Received 2 April 2019; published 13 November 2019)}

\begin{abstract}
A wide variety of complex phases in quantum materials are driven by electron-electron interactions, which are enhanced through density of states peaks. A well-known example occurs at van Hove singularities where the Fermi surface undergoes a topological transition. Here we show that higher order singularities, where multiple disconnected leaves of Fermi surface touch all at once, naturally occur at points of high symmetry in the Brillouin zone. Such multicritical singularities can lead to stronger divergences in the density of states than canonical van Hove singularities, and critically boost the formation of complex quantum phases via interactions. As a concrete example of the power of these Fermi surface topological transitions, we demonstrate how they can be used in the analysis of experimental data on $\mathrm{Sr}_{3} \mathrm{Ru}_{2} \mathrm{O}_{7}$. Understanding the related mechanisms opens up new avenues in material design of complex quantum phases.
\end{abstract}

DOI: 10.1103/PhysRevLett.123.207202

Introduction.-The properties of unconventional phases in quantum materials are generally connected to features of the electronic band structure. For example, in density waves, characteristic wave vectors of emergent order parameters can often be related to nesting-type features of the underlying Fermi surface (FS) as discussed for, e.g., iron pnictides [1], organics [2], and transition metal dichalcogenides [3]. Yet these nesting features in themselves usually cannot account for the observed thermodynamic stability of such correlated quantum phases. Intriguingly, in a range of these materials, the band structure hosts energetically close-by singularities in the density of states $\nu$ (DOS), which have been conjectured often to be crucial ingredients stabilizing the emergent phases.

Singularities in the DOS occur naturally at FS topological Lifshitz transitions (LT). A prominent example is the van Hove singularity (vHs) formed at a saddle point in the energy-momentum dispersion [see Fig. 1(a)]. A twodimensional (2D) vHs has a relatively weak logarithmic divergence in the DOS, but it is known to lead to a wealth of phenomena such as ferromagnetism driven by the Stoner mechanism (see, e.g., Ref. [4]). An important point is that the thermodynamic stability of the emergent phases depends on the magnitude of the singularity as well as its shape [4] (i.e., gradient and curvature). As a consequence stronger power law divergences can have a much more dramatic impact on the formation of complex ordered phases than the weaker vHs.
The identification of the type of these singularities in correlated quantum materials is an important first step to understand their properties, given that many experimental quantities, with puzzling dependencies on the external probes, can be explained in a natural way. Here, we explore the consequences of a generalization of these concepts to multicritical topological transitions where multiple disjoint parts of a FS merge and demonstrate the power of the singularities in explaining properties of the correlated material $\mathrm{Sr}_{3} \mathrm{Ru}_{2} \mathrm{O}_{7}$.

Multicritical FS topological transitions naturally occur at points of high crystal symmetry, where the number $n$ of FS components merging depends on the particular symmetry. In Figs. 1(a)-1(f) we illustrate the symmetries associated with the $n=2$ (vHs), 3 and 4 cases in 2D. When the singularity occurs at an edge of a Brillouin zone (BZ) there are generically two pieces $(n=2)$ of the FS that join at the singularity, as depicted in Fig. 1(a). At the critical energy (dotted line) there is a topology change in the FS structure with $n=2$ FSs touching and reconnecting. At the corner of a hexagonal BZ, three FSs or leaves $(n=3)$ can join at the singularity, as happens in, e.g., biased bilayer graphene [5], [Figs. 1(c) and 1(d)]. In the square lattice, four leaves $(n=4)$ can meet at the $X$ point in the corner of the BZ [Fig. 1(f)]. At these high symmetry points higher order terms in the dispersion can become relevant, critically changing the divergence of the DOS, e.g., from a logarithmic to a stronger power-law divergence in the $n=4$ case discussed below. 
(a) $\mathrm{Sr}_{2} \mathrm{RuO}_{4}$

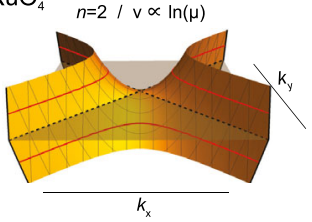

(c) Bilayer

Graphene

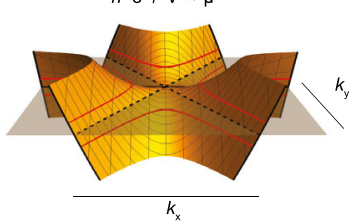

(d)
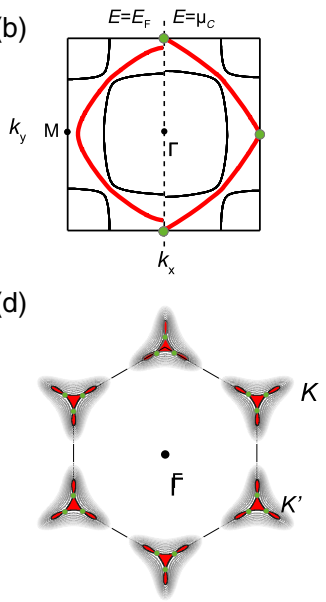

(e) $\mathrm{Sr}_{3} \mathrm{Ru}_{3} \mathrm{O}_{7}$
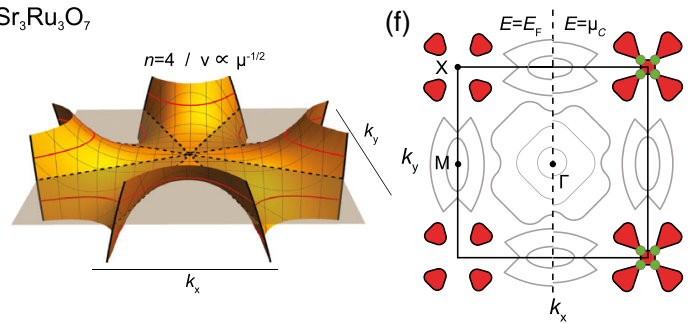

FIG. 1. (a) The $n=22 \mathrm{D}$ van Hove singularity in the form of a saddle point. The DOS $\nu$ diverges as $\ln \mu$. Red lines indicate FSs above and below the singularity. The critical FS is shown by the dotted black line. (b) This singularity occurs for example very close to the Fermi energy in the case of $\mathrm{Sr}_{2} \mathrm{RuO}_{4}$ (green marker for the critical chemical potential) (c) The $n=3$ singularity that can occur at threefold symmetric points. (d) The band structure or FS of bilayer graphene that is very close to such a $n=3$ monkey saddle singularity. (e) The $n=4$ singularity at a fourfold symmetric point. (f) Schematic of the quasi-2D FS of $\mathrm{Sr}_{3} \mathrm{Ru}_{2} \mathrm{O}_{7}$ in the $k_{z}=0$ plane at the Fermi energy (left side) and at $\mu_{C}$ (right side). The crucial bands that are close to the $n=4$ multicritical point are highlighted in red. The central pocket is a small perturbation (see main text). In order to emphasize the characteristic clover leaf FS we show an extended $k$-space picture beyond the BZ boundaries.

An explicit illustration of the experimental effects of these concepts is provided by examining the physics of the layered perovskite $\mathrm{Sr}_{3} \mathrm{Ru}_{2} \mathrm{O}_{7}$, which has been intensely studied because of its unusual magnetic and transport properties [6]. We identify a strong power-law singularity at a fourfold symmetric point in momentum space [Fig. 1(f)] and demonstrate how, in conjunction with other features of the FS and electron interactions, this multicritical feature is pivotal for the physical properties of this material, explaining several previously perplexing characteristics. This is the first tangible demonstration in an existing layered material of the effects of such multicritical LTs (MLTs).

Effective dispersion relation and analysis for $n=4$.The energy-momentum dispersion relation in the vicinity of a $n=4$ MLT can be approximated by the simple expression

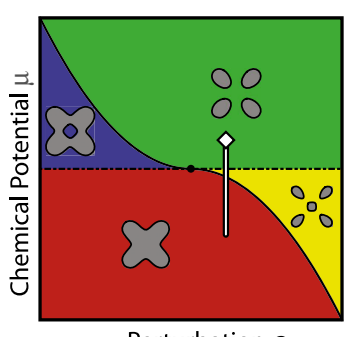

Perturbation $a$

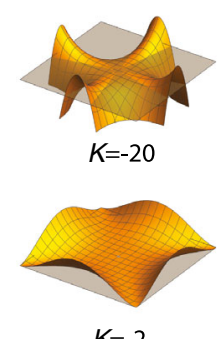

$K=-2$

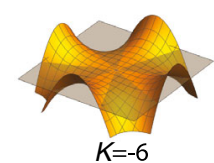

$K=-6$

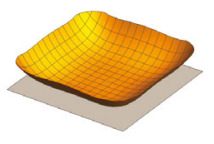

$K=-1 / 2$
FIG. 2. Schematic phase diagram of the relevant hole-like version of the effective dispersion relation (1). In $\mathrm{Sr}_{3} \mathrm{Ru}_{2} \mathrm{O}_{7}$ there is no control over the parameter $a>0$, so that only red, yellow, and green phases are accessible. There are two lines of LTs, a dashed-dotted line of a band edge type and a solid line corresponding to the transition of the neck-narrowing type. The $X_{9}$ singularity is located at the crossing of these two lines. The white line schematically shows the location of $\mathrm{Sr}_{3} \mathrm{Ru}_{2} \mathrm{O}_{7}$ within this phase diagram with the diamond marking the location in zero field. (Right side) The three dimensional surfaces above are electron dispersions $\varepsilon=\varepsilon\left(k_{x}, k_{y}\right)$ in the vicinity of the singularity. The gray horizontal plane represents the critical energy of the singularity $\varepsilon=0$.

$$
\varepsilon(\vec{k})=\left\{\begin{array}{l}
a k^{2}+k^{4} \cos 4 \varphi-\mu, \\
a\left(k_{x}^{2}+k_{y}^{2}\right)+\left(k_{x}^{4}-6 k_{x}^{2} k_{y}^{2}+k_{y}^{4}\right)-\mu,
\end{array}\right.
$$

where the first line is a representation in polar coordinates (with $\varphi$ being the azimuthal angle) and the second being a representation in Cartesian coordinates.

For a small, nonzero, positive $a$ this dispersion exhibits two LTs as we change the chemical potential $\mu$ (see Fig. 2). At smaller values of $\mu$ one large holelike FS exists. At the critical chemical potential four vHs appear at a FS topological transition where a new center pocket is created. As the chemical potential is increased further, the FS undergoes a second LT, of the band edge type, with the vanishing of the center pocket.

If the dispersion relation can be tuned closer to $a=0$, then the singularity is approached and the vHs merge with the minimum of the central electron pocket to form a fourth order saddle:

$$
4 \times \underbrace{\left(k_{x}^{2}-k_{y}^{2}\right)}_{\text {vH saddle }}+1 \times \underbrace{k^{2}}_{\text {e/h pocket }} \leftrightarrow \underbrace{k^{4} \cos 4 \varphi}_{\text {4th order saddle }} .
$$

The singularity can be viewed as a Lifshitz multicritical point, as it appears at the crossing of two LTs and sits at the border of four different topological phases, see Fig. 2. Such behavior can be described within the framework of singularity theory [7] by a symmetry-restricted unimodal parabolic singularity $X_{9}$ in the electron dispersion $\varepsilon(\vec{k})$. The core of the singularity is the fourth order terms, generalized to $k_{x}^{4}+K k_{x}^{2} k_{y}^{2}+k_{y}^{4}$ in Cartesian coordinates. This term is the germ of the singularity, while the remaining terms $\left[a\left(k_{x}^{2}+k_{y}^{2}\right)-\mu\right]$ represent the perturbation unfolding the 
singularity. Unlike simpler singularities, $X_{9}$ forms a whole family of singularities parametrized by the modulus $K$. While a generic singularity from the $X_{9}$ family has a codimension of eight, one modulus and seven control parameters, the presence of the lattice symmetry greatly simplifies the situation leaving only the modulus $K$ and two control parameters $a, \mu$. The consequences of the singularity on physical properties are the same for the whole range $K<-2$. The value of the modulus $K=-6$ is special as it corresponds to electronlike and holelike sections of the same width, a property that is confirmed in the DFT calculation. This implies existence of an additional symmetry in the system, a superposition of the particle-hole transformation $\varepsilon \leftrightarrow-\varepsilon$ and rotation by an angle $\pi / 4$. If we increase the value to $K=-2$, then the system reaches a critical point and the saddle disappears, leaving a singular $\propto k^{4}$ electronic pocket. All values of the modulus $K<-2$ lead to the same topological features.

In the relevant parameter regime $K<-2$ the DOS of this dispersion has a critical $\propto|\mu|^{-1 / 2}$ scaling for $a=0$ and can be summarized as

$$
\nu(\mu) \propto \begin{cases}\left|\mu-\mu_{c}\right|^{-1 / 2}, & |\mu| \gg \mu_{c}, \\ \ln \frac{\mu_{c}}{\left|\mu-\mu_{c}\right|}, & \left|\mu-\mu_{c}\right| \ll \mu_{c},\end{cases}
$$

where the critical value of the chemical potential $\mu_{c}=$ $a|a| / 4$ (more details in the Supplemental Material [8]).

Experimental consequences of the singularities in correlated systems: $\mathrm{Sr}_{3} \mathrm{Ru}_{2} \mathrm{O}_{7}$. - There are profound consequences of this singularity for $\mathrm{Sr}_{3} \mathrm{Ru}_{2} \mathrm{O}_{7}$, a material of wide interest due to the observed phenomena in the vicinity of a metamagnetic quantum critical end point (QCEP) at $H_{c}=8 \mathrm{~T}$ for fields parallel to the crystallographic $c$ axis [26,27].

In Fig. 3 the emergent phase diagram is schematically shown highlighting several features relevant for our discussion (for a review [6]). Approaching $H_{c}$ as a function of $T$, a logarithmic divergence in specific heat divided by temperature $C / T$ is observed $[28,29]$. The approach to $H_{c}$ as a function of magnetic field in the Fermi liquid (FL) regime is characterized by a singular contribution to $C / T$. Careful analysis [30] reveals a power law divergence of $C / T$ as a function of reduced field $h=\left(H-H_{C}\right) / H_{C}$ with an unexpected exponent of $(-1)$. It has been suggested that the singularities in $C / T$ as a function of field or temperature are consistent with a 2D QCEP point within the canonical description of quantum criticality [31]. The expected exponent within this theoretical framework is $-1 / 3$ and in general has to be fixed in any fit of this model [32]. The observed exponent of $(-1)$ in an assumption-free power law fit to $C / T$ therefore posed important theoretical questions.

At low temperature, access to the QCEP is preempted by an unusual set of emergent phases (labeled $A$ and $B$ in Fig. 3) [33,34]. Neutron scattering measurements [33]

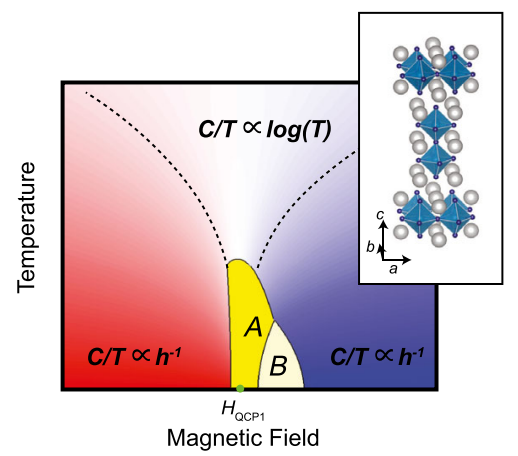

FIG. 3. Schematic phase diagram of $\mathrm{Sr}_{3} \mathrm{Ru}_{2} \mathrm{O}_{7}$ together with the crystal structure shown in the inset (see Ref. [6] for more details).

revealed an incommensurate magnetic order with a wave vector $\vec{Q}^{A}=( \pm 0.233,0,0) /(0, \pm 0.233,0)$ and $\vec{Q}^{B}=$ $( \pm 0.218,0,0) /(0, \pm 0.218,0)$, respectively.

Fermi surface as calculated by density functional theory.-To understand the origin of the singularity in the DOS we performed density functional theory (DFT) calculations $[8,35,36]$. The calculated band structure for zero magnetization agrees broadly with ARPES data [37,38] [Fig. 4(a)]. While the chemical potential is slightly higher than observed experimentally, this does not affect the main conclusions drawn here. We therefore consider the evolution of the DOS with increasing magnetic moment per unit cell (see Fig. 4), as a convenient way to model the effects of an applied magnetic field. By increasing the magnetic moment a LT is observed at the $X$ point at a magnetization of around $0.5 \mu_{B} / \mathrm{Ru}$. This LT dominates the DOS and the thermodynamic properties. In order to (a)

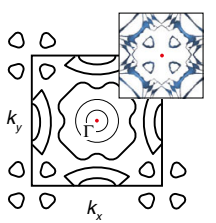

(d)

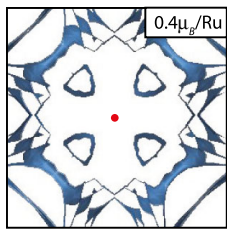

(b)

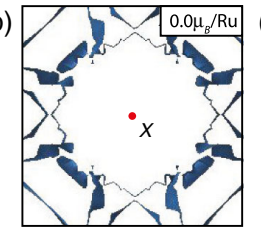

(e)

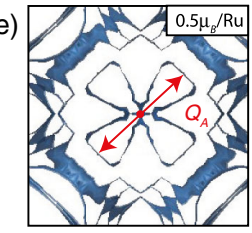

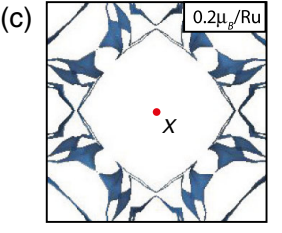

(f)

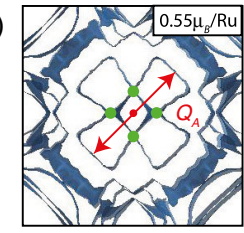

FIG. 4. Result of the DFT calculation. (a) Here we show the schematic FS structure from Fig. 1(e) together with the $k_{z}$ projected DFT calculation to aid orientation. (b)-(f) Projected FSs for values of magnetization $\mu=0.0,0.2,0.4,0.5,0.55 \mu_{B}$ per unit cell as calculated by the DFT and centered at the $X$ point. The topological transitions are evident for the value of magnetization close to 0.5 and $0.55 \mu_{B}$ per unit cell. The red arrow in (e), (f) connects the two nested parts of the characteristic clover leaf structure giving rise to the density wave in the $A$ phase. 
identify the essential requirements generating the key FS features we derive a quasi-2D tight binding model based on the $\mathrm{Ru}$ 4D orbitals (see the Supplemental Material [8]) relevant at the Fermi energy [39] and adjusted to accurately describe the relevant part of the ARPES data. The resulting FS is shown in Fig. 1(f). A careful study of the band structure reveals that it is well described by an effective dispersion given in Eq. (1). The DFT calculations suggest the value of $K$ close to $K=-6$, implying an effective power law divergence [Eq. (3)].

Qualitatively similar conclusions can be drawn from the tight binding model which unveils the microscopic origin of the MLT point (see the Supplemental Material [8]). In the aristotype structure without $\mathrm{RuO}_{2}$ octahedra rotations the band structure exhibits a $n=2 \mathrm{vHs}$ at an $M$ point of the BZ. Counterintuitively the rotations, while lowering the crystal symmetry, reconstruct the BZ such that the singularity is transformed into an $n=4$ MLT point at an $X$ point in the new BZ. This is an important guiding principle how to stabilize such LT.

The characteristic clover leaf structure of the FSs in the vicinity of the LT naturally gives rise to strong nesting of the edges of the $\gamma$ bands which, in combination with the MLT, helps generate the SDW. It is important to note that the nested FS parts have a distinct orbital character $\left(d_{x z / y z}\right)$ from those FS parts that create the MLT $\left(d_{x y}\right)$. The value of the nesting vector $\vec{Q}=( \pm 0.23,0,0)$ or $(0, \pm 0.23,0)$ is effectively that which is observed experimentally in phase $A$. These nested parts of the FS determine the wavelength of the observed SDW (see also [33]) within our theoretical model.

Magnetic field approach to criticality.-The MLT has a profound effect on the specific heat $C_{v}$ as a function of the magnetic field $\left(C_{v} \propto\left|H-H_{c}\right|^{-1}\right)$. The power-law divergence of the DOS as a function of energy leads to the divergence of $C_{v}$ with the field [30]. $C_{v}$ is determined by the value $\nu\left(\epsilon_{F}\right)$ of the DOS at the Fermi level: $C_{v}=$ $(\pi / 2) k_{B} T \nu\left(\epsilon_{F}\right)$. Near the singularity $\nu(\epsilon) \propto\left|\epsilon-\epsilon_{c}\right|^{-1 / 2}$, where $\epsilon_{c}$ is the location of the singularity. Including magnetic field to lowest order, the DOS is $\nu(\epsilon, H)=$ $\frac{1}{2}\left[\nu\left(\epsilon+g \mu_{B} H\right)+\nu\left(\epsilon-g \mu_{B} H\right)\right]$. Charge conservation requires the Fermi energy to shift nonlinearly with $H$ :

$$
\begin{aligned}
& {\left[\epsilon_{F}(H)-\epsilon_{c}+g \mu_{B} H\right]^{1 / 2}+\left[\epsilon_{F}(H)-\epsilon_{c}-g \mu_{B} H\right]^{1 / 2}} \\
& \quad=2\left[\epsilon_{F}(H=0)-\epsilon_{c}\right]^{1 / 2}
\end{aligned}
$$

At $H_{c}, \epsilon_{F}\left(H_{c}\right)=\epsilon_{c}+g \mu_{B} H_{c}$ as the singularity is within the minority band. Then $\epsilon_{F}(0)-\epsilon_{c}=\frac{1}{2} g \mu_{B} H_{c}$ and for $H$ near $H_{c}$ Eq. (4) reads $\epsilon_{F}(H)=\epsilon_{c}+g \mu_{B}\left(H^{2}+H_{c}^{2} / 2 H_{c}\right)$. Therefore, $\nu\left[\epsilon_{F}(H)\right] \propto 1 /\left|H-H_{c}\right|$ and the specific heat as well as the entropy is proportional to $1 /\left|H-H_{c}\right|$. This explains the experimental data and is a direct fingerprint of the existence and the importance of the $n=4$ MLT in $\mathrm{Sr}_{3} \mathrm{Ru}_{2} \mathrm{O}_{7}$.
Phase formation.-As explained, there is significant nesting along $\vec{Q}^{A}$ in the $\gamma$ band giving rise to a susceptibility to SDW formation. As the SDW and the MLT originate from different orbitally orthogonal parts of the FS, at tree level in a renormalization group (RG) sense, the two processes can be treated as decoupled. At a higher RG order, this is no longer true and the DOS singularity influences the thermodynamic stability of the SDW. The mechanism of this particular LT as described above involves the creation of a pocket at the $X$ point. An effect of interactions is that this pocket formation is a first order transition with a jump to a higher total number of fermions in the relevant bands [40]. This is consistent with experimental observations upon entering the $A$ phase as a function of field. The additional pocket, in combination with the MLT point, provides additional FS degrees of freedom. This leads to the counterintuitive result of the high-field $A$ phase having a higher entropy than the low field FL phase, as is established experimentally [30].

Temperature approach to criticality.-The logarithmic divergence $C_{v} \propto T \log (1 / T)$ [Fig. 1(d)] as a function of temperature is clearly an effect of interactions and a sign of quantum criticality. As shown previously [41], the formation of a small pocket in the middle of a larger FS leads to the same result due to interactions. Alternatively, it can be thought as a consequence of the scattering of "light" electrons further from the singularity, off "heavy" electrons [42]. In addition, a correlated 2D system with self-energy which is position dependent ( $k$ dependent) leads to the same behavior $[43,44]$. In the case of SDW formation, the self-energy correction is $\Sigma(\omega, \vec{k}) \propto \omega / k$ (with $k=k_{\|}$), giving an effective mass $m^{*}=m\left[1-\partial \Sigma /\left.\partial \omega\right|_{\omega \rightarrow 0}\right]$. Therefore, given that $C_{v} \propto T \int m^{*} d k$, then naturally $C_{v} \propto T \log (1 / T)$. These conditions are fundamentally linked to the MLT, leading to qualitatively similar behavior.

Discussion.-The effects of simple LTs were explored in several classes of quantum materials (e.g., [41,45-48]). In this work, we have demonstrated how an MLT is formed at a high symmetry point in the BZ can lead to a wealth of unusual physical phenomena. This was illustrated concretely through the example of $\mathrm{Sr}_{3} \mathrm{Ru}_{2} \mathrm{O}_{7}$, in which an MLT happens in the $\gamma$ bands at the $X$ point of the BZ, leading to a four-leaf $(n=4) \mathrm{FS}$. This is accompanied by a large peak in the DOS, describable by a divergence as an inverse square root in energy singularity. We showed how this power-law in combination with the emergent central pocket and enhanced spin or charge susceptibilities due to independent, orbitally orthogonal parts of the FS are consistent with a wide range of previously puzzling experimental data. The intriguing behavior of this well-characterized material is explained within the framework of higher singularities. For any $n=4$ singularity in quasi-2D materials the phase diagram presented in Fig. 2 is relevant, and whenever $K<-2$ a regime of effective power-law divergence should appear. 
The example of $\mathrm{Sr}_{3} \mathrm{Ru}_{2} \mathrm{O}_{7}$ demonstrates one important finding; the FL parameters at zero field are extremely robust against tuning over a much wider range than previously believed. A direct implication would be that quantum fluctuation corrections are not relevant over a wide region of magnetic fields in the FL regime of the phase diagram. For example, significant further band renormalization might not be expected. This is not only surprising but should also lead to a careful reevaluation of other materials. While the singularity dominates the thermodynamics, the heavy quasiparticles only indirectly contribute to transport through the scattering of electrons from other parts of the FS off them. Therefore, there is a subtle interplay of the importance of each part of the FS to different experiments.

$\mathrm{Sr}_{3} \mathrm{Ru}_{2} \mathrm{O}_{7}$ serves as a model system and guide to a whole range of material classes in which MLTs occur (see the Supplemental Material [8] for a discussion on generic mechanisms in, e.g., ruthenates [49], bilayer graphene [5,46,50,51], and transition metal dichalcogenides [52]). One key lesson is that the nontrivial divergences in the DOS are a key driver in thermodynamically stabilizing unconventional phases, originating from otherwise independent (orbitally orthogonal) parts of the FS. The achievement of this work is to identify the importance of the MLT and thereby help to disentangle the roles of the LT in the band structure on the one hand and (quantum) fluctuations and interactions on the other. In all quasi-2D materials with an $n=4$ singularity the phase diagram presented in this work is relevant and whenever $K<-2$ a regime of powerlaw divergence should appear. The counterintuitive mechanism that turns a trivial $n=2 \mathrm{vHs}$ into a $n=4$ MLT by lowering the crystal symmetry, provides a new guiding principle how to stabilize complex quantum phases.

We thank F. Baumberger, C. Castelnovo, A. Chubukov, G. Goldstein, C. Hicks, and Y. Sherkunov for helpful discussions. The work was supported by the EPSRC Grants No. EP/P002811/1 (JJB) and No. EP/P024564/1 (AWR), the Royal Society (JJB and CC) and the DOE Grant No. DE-FG02-06ER46316 (CC).

*These two authors contributed equally.

a.rost@st-andrews.ac.uk

*J.Betouras@lboro.ac.uk

[1] P. Dai, J. Hu, and E. Dagotto, Nat. Phys. 8, 709 (2012).

[2] P. Monceau, Adv. Phys. 61, 325 (2012).

[3] A. M. Gabovich, A. I. Voitenko, and M. Ausloos, Phys. Rep. 367, 583 (2002).

[4] E. P. Wohlfarth and P. Rhodes, Philos. Mag. 7, 1817 (1962).

[5] A. Shtyk, G. Goldstein, and C. Chamon, Phys. Rev. B 95, 035137 (2017).

[6] A. P. Mackenzie, J. A. N. Bruin, R. A. Borzi, A. W. Rost, and S. A. Grigera, Physica (Amsterdam) 481C, 207 (2012).
[7] V. I. Arnold, Singularity Theory, London Mathematical Society Lecture Note Series Vol. 53 (Cambridge University Press, Cambridge, England, 1981).

[8] See Supplemental Material at http://link.aps.org/ supplemental/10.1103/PhysRevLett.123.207202 for details, which also includes Refs. [9-25].

[9] S.-I. Ikeda, Y. Maeno, S. Nakatsuji, M. Kosaka, and Y. Uwatoko, Phys. Rev. B 62, R6089 (2000).

[10] H. Yaguchi, R. S. Perry, and Y. Maeno, AIP Conf. Proc. 850, 1203 (2006).

[11] A. M. Berridge, A. G. Green, S. A. Grigera, and B. D. Simons, Phys. Rev. Lett. 102, 136404 (2009); A. M. Berridge, S. A. Grigera, B. D. Simons, and A. G. Green Phys. Rev. B 81, 054429 (2010).

[12] J. Lee, M. P. Allan, M. A. Wang, J. Farrell, S. A. Grigera, F. Baumberger, J. C. Davis, and A. P. Mackenzie, Nat. Phys. 5, 800 (2009).

[13] J. F. Mercure et al., Phys. Rev. B 81, 235103 (2010).

[14] H. Shaked, J. D. Jorgensen, O. Chmaissem, S. Ikeda, and Y. Maeno, J. Solid State Chem. 154, 361 (2000).

[15] L. Zhu, M. Garst, A. Rosch, and Q. Si, Phys. Rev. Lett. 91, 066404 (2003).

[16] M. H. Fischer and M. Sigrist, Phys. Rev. B 81, 064435 (2010).

[17] C. Piefke and F. Lechermann, Phys. Status Solidi B 248, 2269 (2011).

[18] C. Autieri, M. Cuoco, and C. Noce, Phys. Rev. B 89, 075102 (2014).

[19] E. Rozbicki, Ph.D. thesis, University of St. Andrews, 2011.

[20] A. M. Berridge, Phys. Rev. B 83, 235127 (2011).

[21] N. Kikugawa, A. W. Rost, C. W. Hicks, A. J. Schofield, and A. P. Mackenzie, J. Phys. Soc. Jpn. 79, 024704 (2010).

[22] A. Charnukha, S. Thirupathaiah, V. B. Zabolotnyy, B. Büchner, N. D. Zhigadlo, B. Batlogg, A. N. Yaresko, and S. V. Borisenko, Sci. Rep. 5, 10392 (2015).

[23] K. Tsutsumi, Phys. Rev. B 26, 5756 (1982).

[24] D. J. Eaglesham, R. L. Withers, and D. M. Bird, J. Phys. C 19, 359 (1986).

[25] Y. Cao et al., Nature (London) 556, 80 (2018).

[26] S. A. Grigera, R. S. Perry, A. J. Schofield, M. Chiao, S. R. Julian, G. G. Lonzarich, S. I. Ikeda, Y. Maeno, A. J. Millis, and A. P. Mackenzie, Science 294, 329 (2001).

[27] S. A. Grigera, P. Gegenwart, R. A. Borzi, F. Weickert, A. J. Schofield, R. S. Perry, T. Tayama, T. Sakakibara, Y. Maeno, A. G. Green, and A.P. Mackenzie, Science 306, 1154 (2004).

[28] R. S. Perry et al., Phys. Rev. Lett. 86, 2661 (2001).

[29] A. W. Rost, S. A. Grigera, J. A. N. Bruin, R. S. Perry, D. Tian, S. Raghu, S. A. Kivelson, and A. P. Mackenzie, Proc. Natl. Acad. Sci. U.S.A. 108, 16549 (2011).

[30] A. W. Rost, R. S. Perry, J. F. Mercure, A. P. Mackenzie, and S. A. Grigera, Science 325, 1360 (2009).

[31] Y. Tokiwa, M. Mchalwat, R. S. Perry, and P. Gegenwart, Phys. Rev. Lett. 116, 226402 (2016).

[32] D. Sun, A. W. Rost, R. S. Perry, A. P. Mackenzie, and M. Brando, Phys. Rev. B 97, 115101 (2018).

[33] C. Lester, S. Ramos, R. S. Perry, T. P. Croft, R. I. Bewley, T. Guidi, P. Manuel, D. D. Khalyavin, E. M. Forgan, and S. M. Hayden, Nat. Mater. 14, 373 (2015). 
[34] R. A. Borzi, S. A. Grigera, J. Farrell, R. S. Perry, S. J. S. Lister, S. L. Lee, D. A. Tennant, Y. Maeno, and A. P. Mackenzie, Science 315, 214 (2007).

[35] K. Koepernik and H. Eschrig, Phys. Rev. B 59, 1743 (1999).

[36] http://www.fplo.de.

[37] A. Tamai et al., Phys. Rev. Lett. 101, 026407 (2008).

[38] M. P. Allan et al., New J. Phys. 15, 063029 (2013).

[39] C. M. Puetter, J. G. Rau, and H.-Y. Kee, Phys. Rev. B 81, 081105(R) (2010).

[40] S. Slizovskiy, J. J. Betouras, S. T. Carr, and J. Quintanilla, Phys. Rev. B 90, 165110 (2014).

[41] S. Slizovskiy, A. V. Chubukov, and J. J. Betouras, Phys. Rev. Lett. 114, 066403 (2015).

[42] E. Berg, S. Hartnoll, and C.H. Mousatov, arXiv: 1810.12945.

[43] S. A. Hartnoll, D. M. Hofman, M. A. Metlitski, and S. Sachdev, Phys. Rev. B 84, 125115 (2011).
[44] Ar. Abanov, A. V. Chubukov, and J. Schmalian, Adv. Phys. 52, 119 (2003).

[45] Y. Sherkunov, A. V. Chubukov, and J. J. Betouras, Phys. Rev. Lett. 121, 097001 (2018).

[46] Y. Sherkunov and J. J. Betouras, Phys. Rev. B 98, 205151 (2018).

[47] A. Ptok, K. J. Kapcia, A. Cichy, A. M. Oleś, and P. Piekarz, Sci. Rep. 7, 41979 (2017).

[48] G. E. Volovik, Low Temp. Phys. 43, 47 (2017).

[49] F. Baumberger et al., Phys. Rev. Lett. 96, 107601 (2006).

[50] Y. Cao, V. Fatemi, S. Fang, K. Watanabe, T. Taniguchi, E. Kaxiras, and P. Jarillo-Herrero, Nature (London) 556, 43 (2018).

[51] N. F. Q. Yuan, H. Isobe, and L. Fu, arXiv:1901.05432.

[52] J. Feng et al., Nano Lett. 18, 4493 (2018). 